\title{
Usefulness of Probe-Based Confocal Laser Endomicroscopy for Esophageal Squamous Cell Neoplasm
}

\author{
Sang Kil Lee \\ Division of Gastroenterology, Department of Internal Medicine, Severance Hospital, Yonsei University College of Medicine, Seoul, Korea
}

See "Bimodal Chromoendoscopy with Confocal Laser Endomicroscopy for the Detection of Early Esophageal Squamous Cell Neoplasms" by Piyapan Prueksapanich, Thanawat Luangsukrerk, Rapat Pittayanon, et al., on page 144-151.

The screening and surveillance program for early detection of esophageal squamous cell carcinoma (ESCC) is essential because advanced ESCC has a poor prognosis. Endoscopic screening with narrow band imaging (NBI) or Lugol chromoendoscopy (LCE) has allowed ESCC diagnosis at an early stage in high-risk patients. ${ }^{1}$ LCE has been the preferred method for long. However, there are several problems due to the use of chemicals in Lugol's iodine, including mucosal irritation and damage of the esophagus and stomach, leading to chest pain, discomfort, and hypersensitivity. LCE has a high sensitivity but a low specificity for the detection of ESCC, leading to a high false positive rate and the need for unnecessary biopsy. ${ }^{2}$

The accuracy of NBI is similar to that of LCE for the detection of early esophageal lesions for screening of high-risk patients. ${ }^{3}$ The surface vascular pattern can be characterized by observing the intrapapillary capillary loops (IPCL) using magnifying NBI. A closer examination of IPCL using magnifying NBI can significantly improve the diagnostic accuracy of ESCC. ${ }^{4}$

Confocal laser endomicroscopy (CLE) is a new technology

Received: February 19, 2019 Revised: March 12, 2019

Accepted: March 12, 2019

Correspondence: Sang Kil Lee

Division of Gastroenterology, Department of Internal Medicine, Severance Hospital, Yonsei University College of Medicine, 50-1 Yonsei-ro, Seodaemun-gu, Seoul 03722, Korea

Tel: +82-2-2228-1996, Fax: +82-2-393-6884, E-mail: sklee@yuhs.ac ORCID: https://orcid.org/0000-0002-0721-0364

(cc) This is an Open Access article distributed under the terms of the Creative Commons Attribution Non-Commercial License (http://creativecommons.org/ licenses/by-nc/3.0) which permits unrestricted non-commercial use, distribution, and reproduction in any medium, provided the original work is properly cited. enabling endoscopists to visualize tissue at the cellular level. ${ }^{5}$ CLE is a technology that enables microscopic visualization $(\times 1,000)$ of the mucosa in real time during endoscopy. CLE can be performed using either via a single endoscope-based system (eCLE; OptiScan, Notting Hill, Australia) or via a probe-based CLE system (pCLE; Cellvizio, Mauna Kea Technologies, Paris, France). ${ }^{6}$ CLE is one of the newest advancements in diagnostic endoscopy and is a highly promising technique for investigating the mucosal surface and the immediate subsurface areas. Cell structures and tissue morphological characteristics can be visualized to a maximum depth of $250 \mu \mathrm{m}$. This technique aims to eliminate the need for biopsies in regions of interest via multiple optical biopsies. The usefulness of CLE is evaluated to shift the focus from random to targeted biopsies. ${ }^{5}$ Previous studies have shown that pCLE has been used to diagnose ESCC with high accuracy., ${ }^{4,-9}$

Compared to LCE or NBI, pCLE cannot be used as a screening method because the area that can be observed is very narrow. pCLE is likely to be applied to distinguish between cancerous and non-cancerous tissue in Lugol unstained lesions or in lesions with NBI abnormalities. pCLE was reported to adequately differentiate between cancerous and non-cancerous lesions in 91.9\% of Lugol unstained lesions. ${ }^{9}$ In this study, the sensitivity and specificity were also high, namely, $94.1 \%$ and $90.0 \%$, respectively.

In the present issue, Prueksapanich et al. ${ }^{10}$ investigated the diagnostic accuracy of dual-focus NBI (dNBI) and LCE combined with pCLE to screen for ESCC. After observation with white light endoscopy, dNBI was carefully performed. Any lesions detected by dNBI were biopsied. Next, LCE was 
performed in the same endoscopic session to detect the $\mathrm{dN}$ BI-missed lesions. Lugol unstained lesions were characterized with pCLE in real time and then biopsied. The histopathological results of the lesions detected by dNBI and the Lugol unstained lesions were considered the gold standard. In this study, dNBI missed high-grade dysplasia (HGD) and lowgrade dysplasia (LGD) in $40 \%$ of the cases. After the dNBI examination, LCE revealed twenty Lugol unstained lesions that were not visible on the prior dNBI. Next, pCLE was used to characterize those Lugol unstained lesions and, nine of the twenty lesions were identified as esophageal squamous cell neoplasms. The sensitivity, specificity, positive and negative predictive values, and accuracy of LCE combined with pCLE in lesions not detected by dNBI were $80.0 \%, 67.0 \%, 44.0 \%$, $91.0 \%$, and $70.0 \%$, respectively.

In this study, pCLE showed lower sensitivity and specificity than those previously reported for the diagnosis of Lugol unstained lesions. ${ }^{9}$ Nevertheless, Prueksapanich et al. argued that pCLE more accurately diagnosed LGD and HGD that were missed by dNBI. ${ }^{10}$ In this study, the authors did not directly compare dNBI with pCLE, and the magnification method differed from that used previously. Therefore, we cannot conclude that pCLE is superior to magnifying NBI in detecting ESCC and its related lesions. Additionally, although Prueksapanich et al. presented pCLE findings of LGD and HGD, there is little evidence that pCLE can accurately diagnose LGD and HGD. $^{10}$

Considering some of the limitations of this study, it seems clear that pCLE has an additional advantage over dNBI for Lugol unstained areas. However, considering that the NBI with magnification method is similar to the LCE method with respect to accuracy, it is difficult to determine whether to use pCLE after LCE or NBI or instead use NBI with magnification. To address this issue, we must compare pCLE with
NBI-magnification or dNBI.

Conflicts of Interest

The author has no financial conflicts of interest.

\section{REFERENCES}

1. Freitag CP, Barros SG, Kruel CD, et al. Esophageal dysplasias are detected by endoscopy with Lugol in patients at risk for squamous cell carcinoma in southern Brazil. Dis Esophagus 1999;12:191-195.

2. Yip HC, Chiu PW. Endoscopic diagnosis and management of early squamous cell carcinoma of esophagus. J Thorac Dis 2017;9(Suppl 8):S689-S696

3. Morita FH, Bernardo WM, Ide E, et al. Narrow band imaging versus lugol chromoendoscopy to diagnose squamous cell carcinoma of the esophagus: a systematic review and meta-analysis. BMC Cancer 2017;17:54.

4. Muto M, Minashi K, Yano T, et al. Early detection of superficial squamous cell carcinoma in the head and neck region and esophagus by narrow band imaging: a multicenter randomized controlled trial. J Clin Oncol 2010;28:1566-1572.

5. Lee SK. Usefulness and future prospects of confocal laser endomicroscopy for gastric premalignant and malignant lesions. Clin Endosc 2015;48:511-515.

6. ASGE Technology Committee. Confocal laser endomicroscopy. Gastrointest Endosc 2014;80:928-938.

7. Liu H, Li YQ, Yu T, et al. Confocal laser endomicroscopy for superficial esophageal squamous cell carcinoma. Endoscopy 2009;41:99-106.

8. Li M, Zuo XL, Yu T, et al. Surface maturation scoring for oesophageal squamous intraepithelial neoplasia: a novel diagnostic approach inspired by first endomicroscopic 3-dimensional reconstruction. Gut 2013;62:1547-1555.

9. Safatle-Ribeiro AV, Baba ER, Faraj SF, et al. Diagnostic accuracy of probe-based confocal laser endomicroscopy in Lugol-unstained esophageal superficial lesions of patients with head and neck cancer. Gastrointest Endosc 2017;85:1195-1207.

10. Prueksapanich P, Luangsukrerk T, Pittayanon R, Sanpavat A, Rerknimitr R. Bimodal chromoendoscopy with confocal laser endomicroscopy for the detection of early esophageal squamous cell neoplasms. Clin Endosc 2019;52:144-151. 